\title{
In vivo knockdown of CXCR4 using jetPEI/CXCR4 shRNA nanoparticles inhibits the pulmonary metastatic potential of B16-F10 melanoma cells
}

\author{
NAYARA DELGADO ANDRÉ ${ }^{1}$, VIVIANE ALINE OLIVEIRA SILVA ${ }^{2}$, CAROLINA BATISTA ARIZA $^{3}$, \\ MARIA ANGELICA EHARA WATANABE ${ }^{3}$ and FERNANDO LUIZ DE LUCCA ${ }^{2}$ \\ ${ }^{1}$ Federal University of São João del-Rei, Divinópolis, Minas Gerais 35501-296; \\ ${ }^{2}$ Department of Biochemistry and Immunology, Ribeirão Preto Medical School, University of São Paulo, Ribeirão Preto, \\ São Paulo 14049-900; ${ }^{3}$ Department of Pathological Sciences, Biological Sciences Center, State University of Londrina, \\ Londrina, Paraná 86057-970, Brazil
}

Received March 24, 2015; Accepted September 25, 2015

DOI: $10.3892 / \mathrm{mmr} .2015 .4487$

\begin{abstract}
Metastasis is a key factor that limits survival in the majority of patients with cancer. Thus, numerous efforts have been made to elucidate the molecular mechanisms involved in this phenomenon. B16-F10 melanoma cells have been demonstrated to be highly metastatic to the lungs in mice. The aim of the current study was to investigate the role of CXC motif chemokine receptor 4 (CXCR4) in the metastatic potential of B16-F10 melanoma cells in mice. In vitro transfection of B16-F10 tumor cells with CXCR4 short hairpin RNA (shRNA) expressing plasmids (CXCR4 shRNA) significantly reduced the expression levels of CXCR4 mRNA (80\%) and protein (68\%), compared with the control. In addition, these results demonstrated that pulmonary metastasis was significantly inhibited (85\%) in mice inoculated with CXCR4 shRNA-transfected B16-F10 melanoma cells. The polycation-based nanoparticle (jetPEI) was used to investigate the effect of CXCR4 knockdown in vivo on the metastatic potential of B16-F10 melanoma cells. The number of pulmonary metastatic nodules was significantly reduced (50\%) in animals that received a retro-orbital injection of jetPEI-CXCR4-1 shRNA. The current study demonstrated that CXCR4 serves a role in the metastatic potential of B16-F10 melanoma cells. Currently there is a great interest in the development of antagonists for the therapeutic targeting of CXCR4 expression. Taking the results of the current study and the fact that CXCR4 is highly conserved between humans and mice into account, this experimental model of metastasis with B16-F10 melanoma cells
\end{abstract}

Correspondence to: Dr Nayara Delgado André, Federal University of São João del-Rei, Divinópolis, Minas Gerais 35501-296, Brazil

E-mail: nayara_fbq@yahoo.com.br

Key words: CXCR4, RNAi, metastasis, B16-F10 melanoma cells, nanoparticles, jetPEI may aid in the discovery of CXCR4 antagonists with clinical implications.

\section{Introduction}

Metastasis is a key factor that limits survival in the majority of patients with cancer. The metastatic potential of malignant melanoma is considerably greater than that of other primary solid tumors (1). The incidence of malignant melanoma has increased during recent decades, with a high mortality rate due to the metastatic dissemination of tumor cells (2).

The molecular mechanisms involved in melanoma metastasis have been thoroughly investigated however remain to be fully understood. It is known that chemokines mediate numerous physiological and pathological processes associated with cell homing and migration (3). The CXC motif chemokine receptor 4 (CXCR4) is a transmembrane receptor that belongs to the chemokine receptor family (4). CXCR4 was initially reported to mediate homing of leukocytes into tissues that produce its ligand, stromal cell-derived factor 1, also known as CXC motif chemokine 12 (CXCL12) $(5,6)$. It has been reported that CXCR4 is the most widely expressed chemokine receptor in numerous different types of cancer, suggesting that this receptor is additionally involved in the migration of tumor cells (2). In this context, it was observed that the expression of CXCL12 is elevated in common sites of metastasis including the lungs, liver, lymph nodes and bone marrow, suggesting that the CXCL12/CXCR4 axis serves a key role in the metastatic destination of numerous types of human cancer $(3,4,7)$.

Numerous previous studies have demonstrated that CXCR4 serves an important role in the metastasis in numerous types of cancer, including human melanoma (1,8-10). It has been reported that CXCL12 binds to CXCR4, which through multiple divergent pathways, leads to cell adhesion, invasion, cell survival and angiogenesis (11-13).

The murine B16-F10 melanoma cell line is highly metastatic to the lungs, and these tumor cells have been used to investigate the molecular mechanisms involved in metastasis $(14,15)$. However, the role served by CXCR4 in this experimental model 
of metastasis remains to be elucidated. Thus, Chung et al (16) observed that the treatment of mice with fucoxanthin inhibits CXCR4, CD44 and matrix metalloproteinase-9 (MMP-9) expression in B16-F10 melanoma cells, which is accompanied by the reduction of lung metastasis. Whilst CD44 and MMP-9 are additionally involved in metastasis, the precise role of CXCR4 in this phenomenon remains unclear in B16-F10 cells. A previous study demonstrated that micro-RNA-199a-5p regulates the expression of 15 metastasis-associated genes in B16-F10 melanoma cells, including CXCR4 (10). However, the current study did not investigate the specific role of CXCR4 in this experimental model of metastasis.

RNA interference (RNAi) is a powerful gene silencing technology with specificity, high efficiency and low toxicity $(17,18)$. Results from phase I human studies are promising, suggesting that RNAi-based cancer therapy may be a potential alternative strategy for the treatment of cancer (19). In a previous study, the role played by the RNA-dependent protein kinase in the growth and metastasis of B16-F10 melanoma cells was investigated using RNAi $(20,21)$.

In the present study, RNAi technology was used to investigate the effect of CXCR4 knockdown on the pulmonary metastasis of B16-F10 melanoma cells in mice. To address this question, B16-F10 melanoma cells were transfected with CXCR4 short hairpin RNA (shRNA)-expressing plasmids, and were subsequently intravenously injected into mice. Additionally, the effect of the intravenous injection of polycation-based nanoparticles (jetPEI/CXCR4 shRNA) on the pulmonary metastasis of B16-F10 melanoma cells was investigated in animals that had previously received an intravenous inoculation of B16-F10 tumor cells. The effects of CXCR4 knockdown in vitro and in vivo were evaluated by counting the number of metastatic nodules in the lungs of mice.

\section{Materials and methods}

Animals. All protocols involving animals were reviewed and approved by the Institutional Animal Care Committee. A total of 200 C57BL/6 mice (6-week-old; 20-25 g; 10 per group) raised at the Central Animal Laboratory of Ribeirão Preto School of Medicine (University of São Paulo; São Paulo, Brazil) were used for the current study. All protocols involving animals were reviewed and approved (certificate no. 062/2006) by the Animal Care Committee of the Ribeirão Preto Medical School.

B16-F10 melanoma cell culture conditions. B16-F10 melanoma cells (Ribeirao Preto Medical School, University of São Paulo, São Paulo, Brazil) were maintained in Roswell Park Memorial Institute 1640 medium (Invitrogen; Thermo Fisher Scientific, Inc., Waltham, MA, USA) supplemented with $10 \%$ inactivated fetal calf serum (Invitrogen; Thermo Fisher Scientific, Inc.), 2 mM L-glutamine (Invitrogen, Thermo Fisher Scientific, Inc.) and $1 \%$ penicillin/streptomycin $(100 \mathrm{U} / \mathrm{ml}$; Gibco; Thermo Fisher Scientific, Inc.) in a humidified atmosphere at $37^{\circ} \mathrm{C}$ and $5 \% \mathrm{CO}_{2}$.

Construction of CXCR4 shRNA expression vector. A total of two shRNA target sequences were selected from different positions within the mouse CXCR4 cDNA sequence (GenBank, accession no. BC031665) corresponding to nucleotides 85-103 (CXCR4-1 shRNA) and 409-427 (CXCR4-2 shRNA). The shRNA sequences are presented in Table I.

These target sequences were BLAST searched (http://blast. ncbi.nlm.nih.gov/Blast.cgi) to ensure that only the CXCR4 gene was targeted. The target sequence of the negative control group used as the control shRNA had no homology with that of humans or mice. The hairpin loop region was annealed with its complementary strand and was cloned into the psiSTRIKE vector controlled by the Pol III U6 promoter (U6 Hairpin Cloning Systems; Promega, Madison, WI, USA) according to the manufacturer's instructions. The screening for the inserts was by digestion with Pst I (Promega).

In vitro transfection. B16-F10 melanoma cells were plated in tissue culture flasks at a density of $7 \times 10^{5}$ cells. Following an overnight incubation and when $\sim 70-80 \%$ confluent, the cells were transfected with $30 \mu \mathrm{g}$ CXCR4 shRNA and $30 \mu \mathrm{l}$ Lipofectamine 2000 (Invitrogen; Thermo Fisher Scientific, Inc.) at a v/w ratio of liposomes to shRNA of 1:1. The transfection efficiency ( $75-80 \%)$ was evaluated using a green fluorescent protein (GFP) expression plasmid (Invitrogen; Thermo Fisher Scientific, Inc.). Prior to the in vivo study, the effectiveness of the two plasmid-based CXCR4-specific shRNAs (CXCR4-1 shRNA and CXCR4-2 shRNA) in reducing the CXCR4 expression in cultured B16-F10 cells was investigated. Subsequently, tumor cells were transfected with CXCR4-1, CXCR4-2 or control shRNA for $5 \mathrm{~h}$, following which the cells were washed, suspended in medium and maintained in culture for 24 or $48 \mathrm{~h}$ at $37^{\circ} \mathrm{C}$. To determine the CXCR4 mRNA and protein levels, lysates of the B16-F10 melanoma cells were used for RNA isolation and western blot analysis.

RNA isolation. Total cellular RNA was extracted using TRIzol-LS Reagent (Invitrogen; Thermo Fisher Scientific, Inc.). The integrity of RNA was assessed using an Agilent Bioanalyzer (Agilent 2100; Agilent Technologies, Inc., Santa Clara, CA, USA).

Analysis of CXCR4 mRNA expression. Reverse transcription-polymerase chain reaction (RT-PCR) was conducted with $1.2 \mu \mathrm{g}$ total RNA and $0.5 \mu \mathrm{g}$ Oligo(dT) primer (Invitrogen; Thermo Fisher Scientific, Inc.), $1 \mathrm{U}$ reverse transcriptase (Promega), 1 U RNase inhibitor (Invitrogen; Thermo Fisher Scientific, Inc.), $5 \mathrm{ml}$ 5X M-MLV RT 5X reaction buffer (Promega) and $4 \mathrm{ml} \mathrm{MgCl}_{2}$ (Promega). The $\beta$-actin gene was used as a reference control gene to normalize the expression value of CXCR4. The primers used for CXCR4 were as follows: Sense, 5'-ACAGGTACATCTGTGACC GCCTTT-3' and anti-sense, 5'-TGCTCTCGAAGTCACATC CTTGCT-3' (Invitrogen; Thermo Fisher Scientific, Inc.; GenBank accession no. BC031665). The oligodeoxynucleotide primers for $\beta$-actin used for amplification were sense 5'-TGGAATCCTGTGGCATCCATGAAAC-3' and anti-sense 5'-TAAAACGCAGCTCAGTAACAGTCCG-3' (Integrated DNA Technologies, Coralville, IA, USA; GenBank Accession No. BC014861) PCR conditions for CXCR4 were as follows: Denaturation for $5 \mathrm{~min}$ at $94^{\circ} \mathrm{C}$ followed by 35 cycles of $1 \mathrm{~min}$ at 
Table I. Sequences of shRNA oligonucleotides.

\begin{tabular}{lcl}
\hline shRNA & Target position & \multicolumn{2}{c}{ Sequence } \\
\hline CXCR4-1 & $85-103$ & 5'-ACCGCGATCAGTGTGAGTATATAAAGTTCTC \\
& & TTATATACTCACACTGATCGCTTTTTC-3' \\
CXCR4-2 & $409-427$ & 5'-ACCGGTAAGGCTGTCCATATCATAAGTTCTCT \\
Control & - & ATGATATGGACAGCCTTACCTTTTC-3' \\
& & 5'-ACCGAAGCGCTGCCGCGACGTTGAAGTTCTCT \\
& & CAACGTCGCGGCAGCGCTTCTTTTC-3'
\end{tabular}

Reference sequences for target positions: BC031665. shRNA, short hairpin RNA; CXCR4, CXC motif chemokine receptor 4.

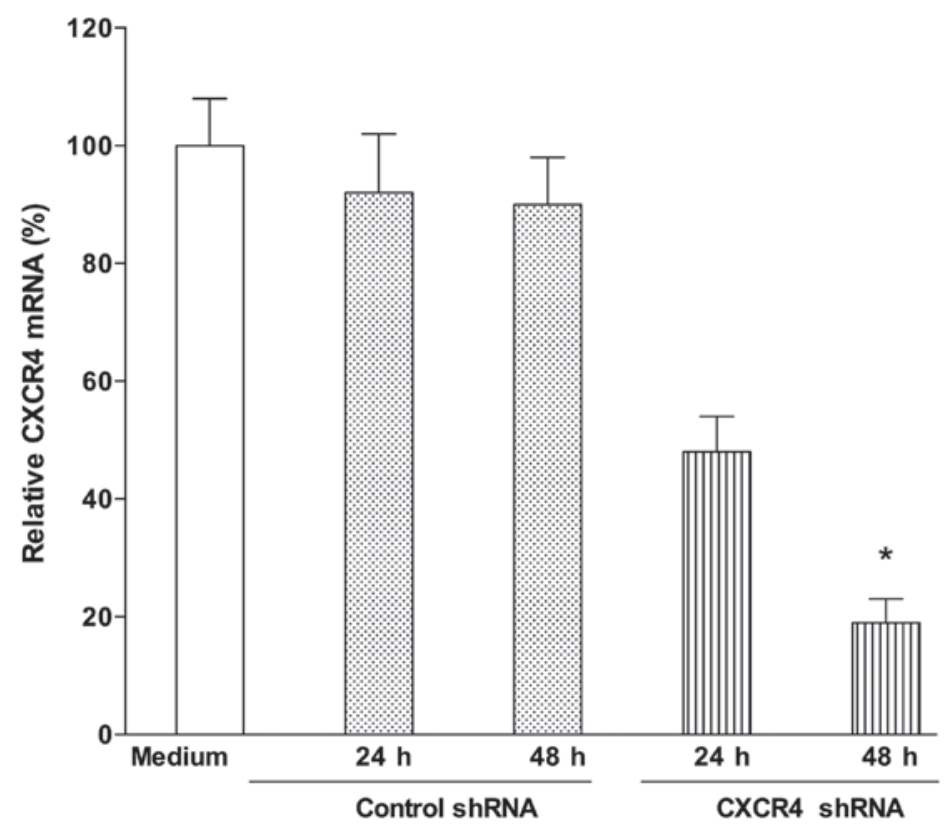

Figure 1. CXCR4 mRNA expression in B16-F10 melanoma cells following transfection with CXCR4 shRNA plasmids. The level of CXCR4 mRNA was determined by reverse transcription-polymerase chain reaction and $\beta$-actin was used as the control. The level of CXCR4 mRNA in B16-F10 melanoma cells incubated with Roswell Park Memorial Institute 1640 medium was set as 100\%. Values are presented as the mean \pm standard deviation of three independent experiments, and the bars are representative of one typical experiment. "P<0.001, vs. control. CXCR4, CXC motif chemokine receptor 4; shRNA, short hairpin RNA.

$94^{\circ} \mathrm{C}, 1 \mathrm{~min}$ at $51^{\circ} \mathrm{C}$ and $1 \mathrm{~min}$ at $72^{\circ} \mathrm{C}$, then 10 min extension at $72^{\circ} \mathrm{C}$ in a thermocycler (PCR-Sprint ThermoHybrid; Abgene; Thermo Fisher Scientific, Inc.). PCR products of $\beta$-actin (364 base pairs) and CXCR4 (291 base pairs) were analyzed by electrophoresis in a $1.5 \%$ agarose gel (Sigma-Aldrich, St. Louis, MO, USA) and visualized using UV fluorescence following staining with ethidium bromide (Merck Millipore, Darmstadt, Germany). Quantification of CXCR4 bands was performed by using ImageQuant software, version 3.3 (GE Healthcare Life Sciences, Chalfont, UK) and the results were expressed as a percentage of the control.

Western blot analysis. B16-F10 adherent cells were detached using ethylenediaminetetraacetic acid (Merck Millipore) and the cell pellet, obtained following centrifugation (1.200 x g for $10 \mathrm{~min}$ at $25^{\circ} \mathrm{C}$ ) was resuspended in $300 \mu \mathrm{l}$ phosphate-buffered saline containing $0.1 \%$ aprotinin, $0.1 \%$ leupeptin and $1 \%$ Triton $\mathrm{X}-100$ (Sigma-Aldrich). Protein concentration was determined by the Cadman method (22). Total cellular protein $(30 \mu \mathrm{g})$ was separated by electrophoresis through 10\% SDS-PAGE (Merck Millipore), and proteins were transferred onto nitrocellulose membranes (GE Healthcare Life Sciences). The membranes were blocked with $10 \%$ dried milk, washed with Tris-buffered saline with $0.1 \%$ Tween-20 for $20 \mathrm{~min}$ and subsequently incubated with 1:250 rabbit anti-CXCR4 polyclonal antibodies (H-118; cat. no. sc-9046; Santa Cruz Biotechnology, Inc., Dallas, TX, USA) for $90 \mathrm{~min}$ at room temperature. Anti-rabbit antibodies conjugated to horseradish peroxidase (GE Healthcare Life Sciences) were added (1:500) and the membranes incubated at room temperature for $60 \mathrm{~min}$ under agitation. Antibody labeled protein bands were visualized using enhanced chemiluminescence detection reagents (GE Healthcare Life Sciences) applied following the manufacturer's instructions. Anti- $\beta$-actin antibody (I-19; cat. no. sc-1616; goat polyclonal; 1,400; Santa Cruz Biotechnology, Inc.) were used as a control. Quantification of bands was performed using ImageQuant software, version 3.3 (GE Healthcare Life Sciences) and the results were expressed as a percentage of the control. 


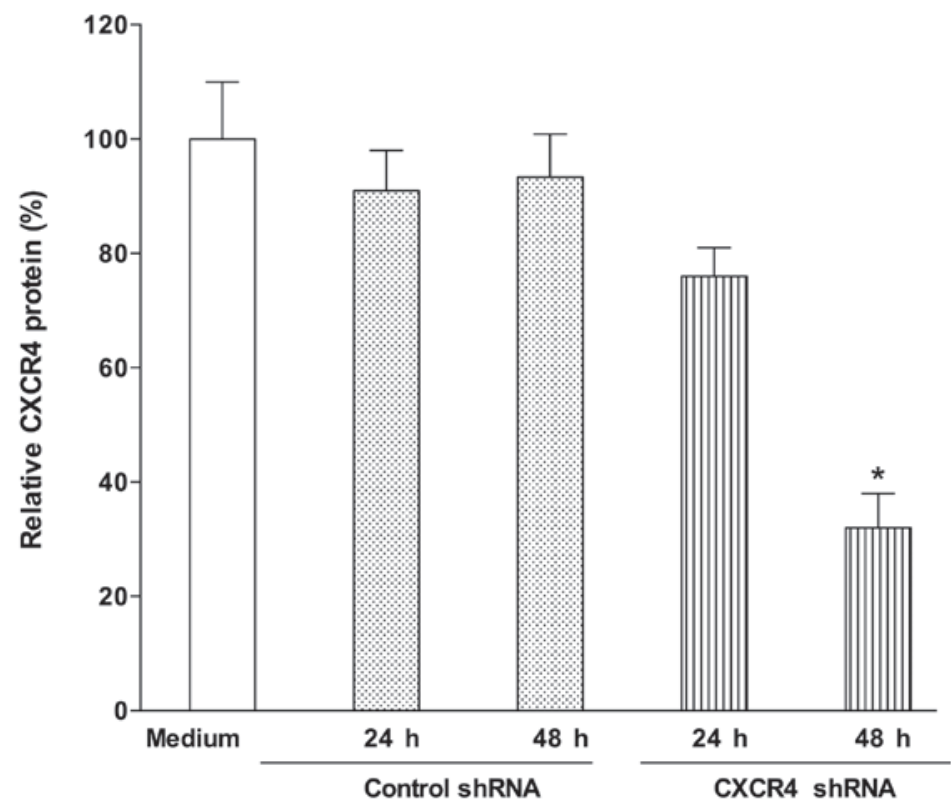

Figure 2. Protein expression levels of CXCR4 in the B16-F10 melanoma cells following transfection with CXCR4 shRNA plasmids, measured by western blot analysis. $\beta$-actin was used as a loading control. The level of CXCR4 protein in B16-F10 melanoma cells incubated with Roswell Park Memorial Institute 1640 medium was set as $100 \%$. Values are presented as the mean \pm standard deviation of three independent experiments, and the bars are representative of one typical experiment. "P<0.001, vs. control. CXCR4, CXC motif chemokine receptor 4; shRNA, short hairpin RNA.

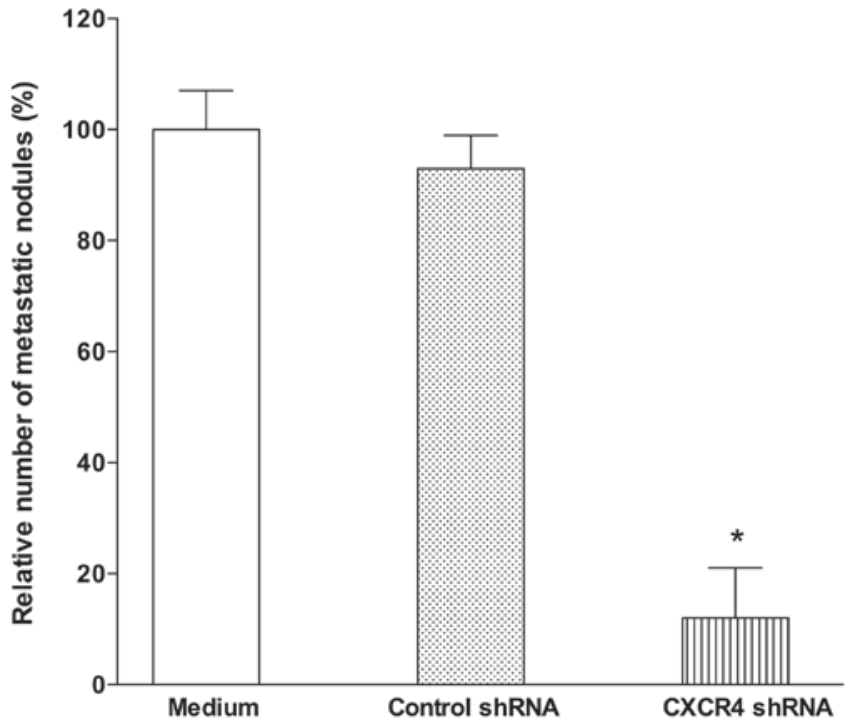

Figure 3. Effect of CXCR4 shRNA on the pulmonary metastatic progression potential of B16-F10 melanoma cells. $n=10$. The animals were sacrificed on day 21 and pulmonary metastatic nodules were counted using a dissecting microscope. Values are presented as the mean \pm standard deviation Data of one representative experiment of three conducted are presented. ${ }^{*} \mathrm{P}<0.001$, vs. control. CXCR4, CXC motif chemokine receptor 4; shRNA, short hairpin RNA.

Retro-orbital injection of transfected B16-F10 melanoma cells into mice. For the in vivo experiments, B16-F10 melanoma cells were transfected with CXCR4-1 shRNA or control shRNA for $5 \mathrm{~h}$, following which the cells were washed in RPMI, suspended in medium (RPMI) and maintained in culture for $24 \mathrm{~h}$ prior to injection into the mice. The viability of cells was assessed by trypan blue staining (Sigma-Aldrich) and was $>95 \%$. The mice were randomly separated into groups of 10 animals. Each group of C57BL/6 mice received by

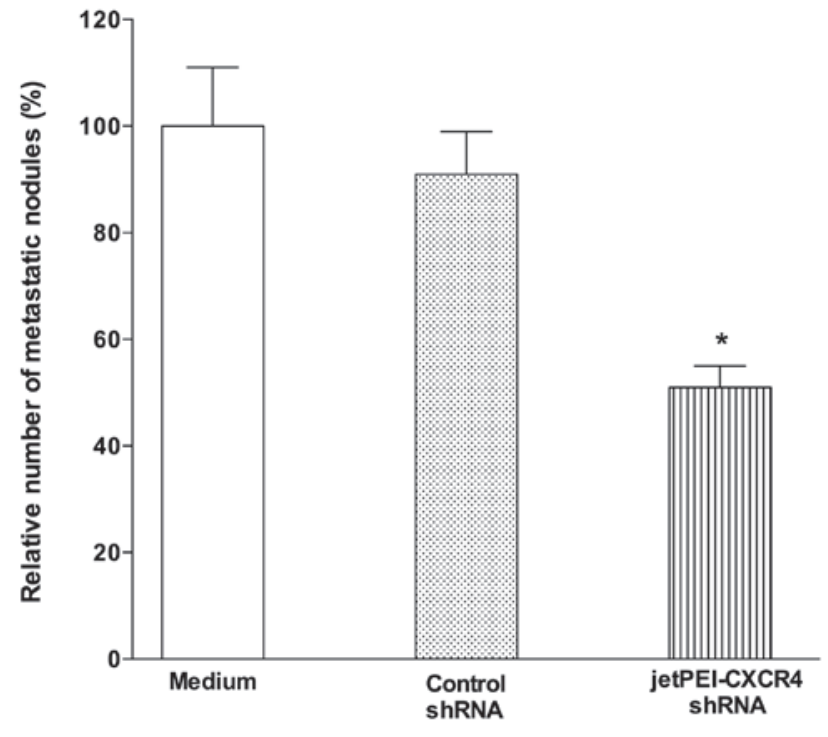

Figure 4. Effect of retro-orbital injection of jetPEI/CXCR4 shRNA on the pulmonary metastatic progression potential of B16-F10 melanoma cells. $\mathrm{n}=10$. Values are presented as the mean \pm standard deviation. Data of one representative experiment of three conducted are presented. ${ }^{*} \mathrm{P}<0.001$, vs. control. CXCR4, CXC motif chemokine receptor 4; shRNA, short hairpin RNA.

intravenous retro-orbital injection, $10^{5}$ tumor cells incubated with medium or transfected with CXCR4-1 shRNA or control shRNA expressing plasmids. On day 21 , mice were sacrificed by cervical dislocation, and the pulmonary metastatic nodules were counted using a dissecting microscope (M80; Leica Microsystems GmbH, Wetzlar, Germany).

Intravenous injection of jetPEI-CXCR4 shRNA. jetPEI ${ }^{\mathrm{TM}}$ is composed of linear polyethylenimine (Polyplus-Transfection SA, Illkirch, France) which ensures effective and repro- 


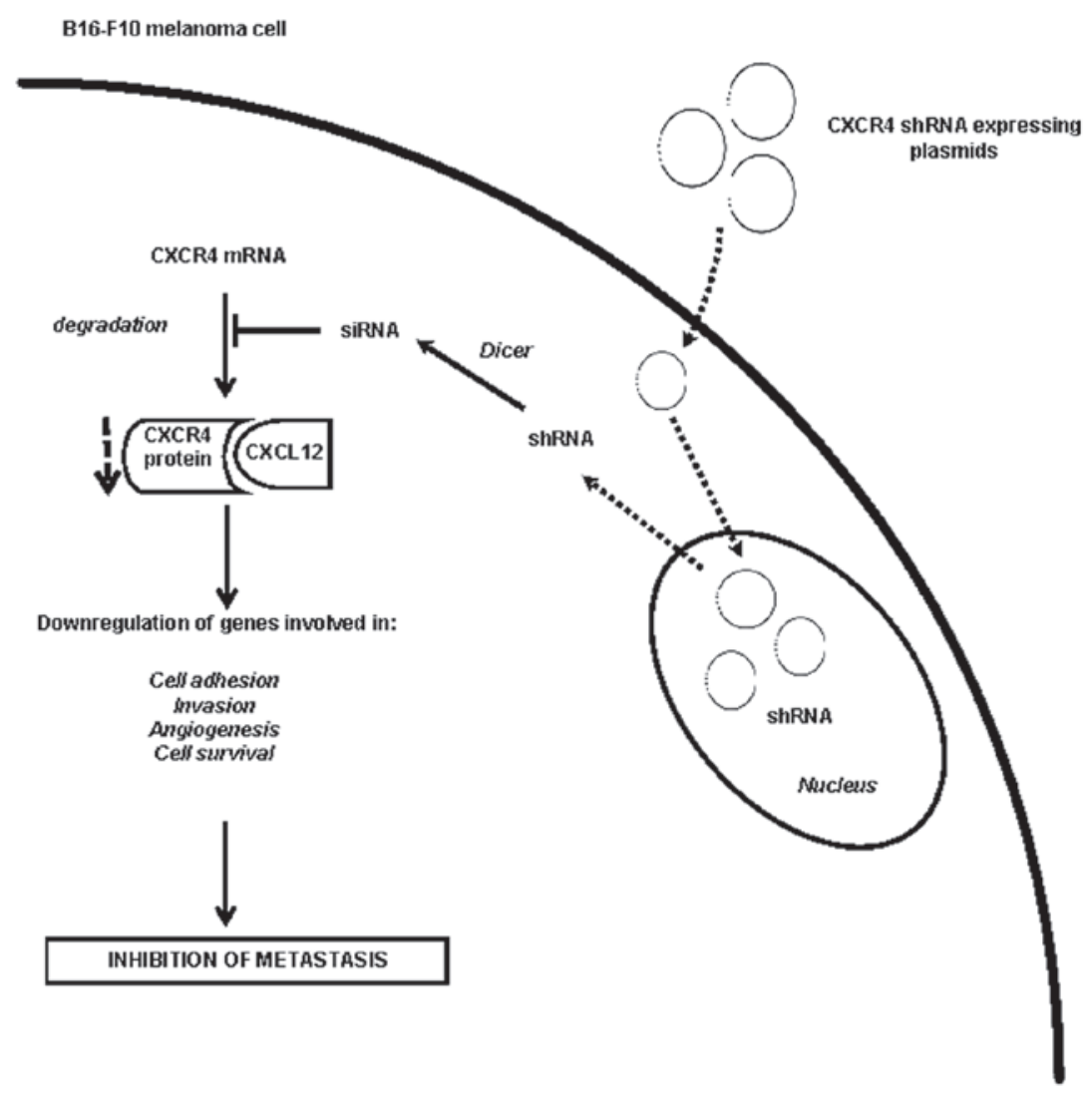

Figure 5. Overview of proposed molecular mechanisms involved in the reduction of pulmonary metastasis following CXCR4 knockdown with CXCR4-shRNA expressing plasmids. CXCR4, CXC motif chemokine receptor 4; CXCL12, CXC motif chemokine 12; shRNA, short hairpin RNA; siRNA, small interfering RNA.

ducible oligonucleotide transfection into mammalian cells with low toxicity. This reagent was a gift from Dr Catherine David (Biotik@ Biotechnologia, São Paulo, Brazil). C57BL/6 mice were inoculated with B16-F10 melanoma cells by retro-orbital injections $\left(10^{5}\right.$ cells/animal $)$. The mice were randomly separated into groups of 10 animals. A total of $10 \mu \mathrm{g}$ plasmid psiSTRIKE (control shRNA and CXCR4 shRNA) was diluted in 10\% glucose solution at a 1:1 ratio and then mixed with jetPEI in the ratio of 1:1 and incubated at room temperature for $20 \mathrm{~min}$. The jetPEI-CXCR4 shRNA was then injected retro-orbitally into the C57BL/6 mice, $12 \mathrm{~h}$ following the inoculation with B16-F10 cells. The mice were sacrificed 21 days following the injection of the tumor cells, and the pulmonary nodules were counted using a dissecting microscope (M80).

Statistical analysis. One-way analysis of variance was used to analyze statistical significance between the groups. Data are presented as the mean \pm standard deviation. Statistical analyses were performed using GraphPad Prism version 5.0 (GraphPad Software, Inc., La Jolla, CA, USA). P $<0.05$ was considered to indicate a statistically significant difference.

\section{Results}

CXCR4 shRNA-expressing plasmids inhibit the expression of CXCR4 mRNA and protein. The ability of the two CXCR4-specific shRNAs expressing plasmid to downregulate
CXCR4 expression in vitro was investigated. B16-F10 melanoma cells were transfected for 24 and $48 \mathrm{~h}$ with the plasmid-based CXCR4-1 shRNA, CXCR4-2 shRNA or control shRNA in the presence of Lipofectamine 2000. Following in vitro transfection, CXCR-4 knockdown was measured by RT-PCR and western blot analysis. The results indicated that the CXCR4-1 shRNA significantly inhibited the expression of CXCR4 mRNA (80\%, P<0.001; Fig. 1) and CXCR4 protein (68\%, $\mathrm{P}<0.001$; Fig. 2) following $48 \mathrm{~h}$ in vitro transfection of tumor cells. Transfection with CXCR4-2 shRNA did not result in a reduction in the mRNA or protein expression of CXCR4 (data not shown). Therefore, CXCR4-1 shRNA was used in all further experiments.

In vitro transfection of B16-F10 melanoma cells with CXCR4 shRNA inhibits pulmonary metastasis. To investigate the effect of reducing CXCR4 expression on the metastatic potential of B16-F10 melanoma cells, the CXCR4-1 shRNA-transfected tumor cells were injected intravenously into mice. Fig. 3 indicates that the pulmonary metastatic nodules were significantly reduced $(\sim 85 \%$; $\mathrm{P}<0.001)$ in mice inoculated with CXCR4-1 shRNA-transfected B16-F10 melanoma cells compared with the control group.

Intravenous injection of jetPEI-CXCR4-1 shRNA inhibits pulmonary metastasis. The jetPEI-CXCR4-1 shRNA was injected retro-orbitally at $12 \mathrm{~h}$ following the inoculation of B16-F10 melanoma cells. Fig. 4 indicates that the 
jetPEI-CXCR4-1 shRNA was able to significantly reduce $(\sim 50 \% ; \mathrm{P}<0.01)$ the number of pulmonary metastatic nodules compared with the control group.

\section{Discussion}

The current study was conducted using B16-F10 melanoma cells, a variant cell line of B16 melanoma, which is highly metastatic to the lungs when injected intravenously into C57BL/6 mice (14). The intravenous injection of B16-F10 melanoma cells via the lateral tail vein is the most commonly used method of administration. However, this method has a high rate of failure and may result in distress due to the animals being placed under a heat lamp to promote peripheral vasodilatation (23). In the present study, retro-orbital injection was observed to be a reliable method for intravascular delivery of B16-F10 melanoma cells compared with injection via the tail vein. This model of metastasis has advantages, including the fact that the metastatic nodules are clearly observable in the lungs due to their high melanin content, and the ease with which the tumor cells may be grown in culture. Furthermore, the B16 murine melanoma is considered a good model of metastasis as it arose spontaneously, and it has been reported that this model accurately recapitulates clinical metastatic melanoma (15). In addition, B16-F10 melanoma cells constitutively express CXCR4 and it is possible to specifically inhibit this chemokine receptor expression through RNAi technology in order to elucidate the possible roles of CXCR4 in metastasis.

The B16-F10 melanoma cells transfected in vitro with CXCR4 shRNA were demonstrated to be effective in inhibiting pulmonary metastasis when intravenously injected into the mice, suggesting that CXCR4 serves a role in this model of metastasis. The next step was to investigate the effect of CXCR4 knockdown in vivo on the metastatic potential of B16-F10 melanoma cells. The instability and inadequate biodistribution of RNAi are key challenges to its clinical use, however nanoparticle formulations have been used to increase the efficacy of RNAi in vivo. Whilst numerous studies have been conducted to optimize nanoparticle gene delivery formulations in vitro, relatively few studies have attempted to deliver genes to experimental tumors in vivo $(24,25)$. The current study used the polycation-based nanoparticle (jetPEI) to investigate the effect of CXCR4-1 knockdown in vivo on the metastatic potential of B16-F10 melanoma cells. This demonstrated that the retro-orbital injection of jetPEI-CXCR4-shRNA significantly reduced the number of pulmonary metastatic nodules. Fidler (14) demonstrated that tumor cells are observed in the lungs of mice shortly following intravenous injection. Furthermore, it was observed that the jetPEI-RNAi nanoparticles are predominantly located in the lung when intravenously injected in mice (26). This suggests that the results of the present study are likely to be due to CXCR4 knockdown in vivo by the jetPEI-CXCR4-shRNA nanoparticles present in the lungs.

The role of the CXCR4/CXCL12 axis in cancer has been extensively investigated. It was observed that CXCR4 is overexpressed in greater than 23 types of human cancer, including melanoma, and contributes to cell proliferation, cell survival, invasion and angiogenesis (3). Based on the results of the current study, the effect of CXCR4 shRNA expressing plasmids may be explained as presented in Fig. 5. Briefly, CXCR4 shRNA expression plasmids were used to transfect B16-F10 melanoma cells in vitro or in vivo with jetPEI-CXCR4-1 shRNA. Following the uptake of CXCR4 shRNA expression plasmids by the tumor cells, CXCR4 shRNA is transcribed in the nucleus, exported to the cytoplasm and processed by dicer to generate CXCR4 siRNA, which induces the specific degradation of CXCR4 mRNA. Therefore, the level of CXCR4 protein is reduced with subsequent downregulation of genes involved in cell survival, cell adhesion, invasion and angiogenesis, resulting in the inhibition of pulmonary metastasis.

The present study demonstrated that CXCR4 serves a role in the formation and progression of pulmonary metastatic nodules in the experimental metastasis model using B16-F10 melanoma cells. However, further studies are required to elucidate the molecular mechanisms involved in this phenomenon. Currently there is interest in the discovery of antagonists for the therapeutic targeting of CXCR4 expression. Considering the results of the current study and the fact that CXCR4 is highly conserved between humans and mice (27), this experimental model of metastasis with B16-F10 melanoma cells may aid in the discovery of CXCR4 antagonists with clinical implications.

\section{Acknowledgements}

The current study was supported by The São Paulo Research Foundation (grant no. 06/57963-1). The authors would like to thank Cacilda D Pereira and Zuleica AS Moraes for their technical assistance.

\section{References}

1. Scala S, Giuliano P, Ascierto PA, Ieranò C, Franco R, Napolitano M, Ottaiano A, Lombardi ML, Luongo M, Simeone E, et al: Human melanoma metastases express functional CXCR4. Clin Cancer Res 12: 2427-2433, 2006.

2. Tsao H, Atkins MB, Sober AJ: Management of cutaneous melanoma. N Engl J Med 351:998-1012, 2004.

3. Chatterjee S, Behnam Azad B and Nimmagadda S: The intricate role of CXCR4 in cancer. Adv Cancer Res 124: 31-82, 2014.

4. Zlotnik A: Chemokines in neoplastic progression. Semin Cancer Biol 14: 181-185, 2004.

5. Zlotnik A, Burkhardt AM and Homey B: Homeostatic chemokine receptors and organ-specific metastasis. Nat Rev Immunol 11: 597-606, 2011.

6. Murdoch C: CXCR4: Chemokine receptor extraordinaire. Immunol Rev 177: 175-184, 2000.

7. Müller A, Homey B, Soto H, Ge N, Catron D, Buchanan ME, McClanahan T, Murphy E, Yuan W, Wagner SN, et al: Involvement of chemokine receptors in breast cancer metastasis. Nature 410: 50-56, 2001.

8. Toyozawa S, Kaminaka C, Furukawa F, Nakamura Y, Matsunaka $\mathrm{H}$ and Yamamoto Y: Chemokine receptor CXCR4 is a novel marker for the progression of cutaneous malignant melanomas. Acta Histochem Cytochem 45: 293-299, 2012.

9. Mitchell B, Leone D, Feller JK, Bondzie P, Yang S, Park HY and Mahalingam M: Correlation of chemokine receptor CXCR4 mRNA in primary cutaneous melanoma with established histopathologic prognosticators and the BRAF status. Melanoma Res 24: 621-625, 2014.

10. Zhou J, Liu R, Wang Y, Tang J, Tang S, Chen X, Xia K, Xiong W, Xu D, Wang S, et al: miR-199a-5p regulates the expression of metastasis-associated genes in B16-F10 melanoma cells. Int J Clin Exp Pathol 7: 7182-7190, 2014.

11. Burger JA and Kipps TJ: CXCR4: A key receptor in the crosstalk between tumor cells and their microenvironment. Blood 107: 1761-1767, 2006. 
12. Duda DG, Kozin SV, Kirkpatrick ND, Xu L, Fukumura D and Jain RK: CXCL12 (SDF1- $\alpha$ ) - CXCR4/CXCR7 pathway inhibition: An emerging sensitizer for anticancer therapies. Clin Cancer Res 17: 2074-2080, 2011.

13. Li Z, Li N, Wu M, Li X, Luo Z and Wang X: Expression of miR-126 suppresses migration and invasion of colon cancer cells by targeting CXCR4. Mol Cell Biochem 381: 233-242, 2013.

14. Fidler IJ: Biological behavior of malignant melanoma cells correlated to their survival in vivo. Cancer Res 35: 218-224, 1975.

15. Bobek V, Kolostova K, Pinterova D, Kacprzak G, Adamiak J, Kolodziej J, Boubelik M, Kubecova M and Hoffman RM: A clinically relevant, syngeneic model of spontaneous, highly metastatic B16 mouse melanoma. Anticancer Res 30: 4799-4803 2010.

16. Chung TW, Choi HJ, Lee JY, Jeong HS, Kim CH, Joo M, Choi JY, Han CW, Kim SY, Choi JS and Ha KT: Marine algal fucoxanthin inhibits the metastatic potential of cancer cells. Biochem Biophys Res Commun 439: 580-585, 2013.

17. Novina CD and Sharp PA: The RNAi revolution. Nature 430: 161-164, 2004.

18. Masiero M, Nardo G, Indraccolo S and Favaro E: RNA interference: implications for cancer treatment. Mol Aspects Med 28 143-166, 2007.

19. Mansoori B, Sandoghchian Shotorbani S and Baradaran B: RNA interference and its role in cancer therapy. Adv Pharm Bull 4: 313-321, 2014.
20. Delgado André N and De Lucca FL: Knockdown of PKR expression by RNAi reduces pulmonary metastatic potential of B16-F10 melanoma cells in mice: Possible role of NF-kappaB. Cancer Lett 258: 118-125, 2007.

21. André ND, Silva VA, Watanabe MA and De Lucca FL: Intratumoral injection of PKR shRNA expressing plasmid inhibits B16-F10 melanoma growth. Oncol Rep 32: 2267-2273, 2014.

22. Cadman E, Bostwick JR, Eichberg J: Determination of protein by a modified Lowry procedure in the presence of some commonly used detergents. Anal Biochem 96(1):21-23, 1979.

23. Yardeni T, Eckhaus $M$, Morris HD, Huizing $M$ and Hoogstraten-Miller S: Retro-orbital injections in mice. Lab Anim 40: 155-160, 2011.

24. Howard KA and Kjems J: Polycation-based nanoparticle delivery for improved RNA interference therapeutics. Expert Opin Biol Ther 7: 1811-1822, 2007.

25. Aigner A: Applications of RNA interference: current state and prospects for siRNA-based strategies in vivo. Appl Microbiol Biotechnol 76: 9-21, 2007.

26. Gao S, Dagnaes-Hansen F, Nielsen EJ, Wengel J, Besenbacher F, Howard KA and Kjems J: The effect of chemical modification and nanoparticle formulation on stability and biodistribution of siRNA in mice. Mol Ther 17: 1225-1233, 2009.

27. Heesen M, Berman MA, Benson JD, Gerard C and Dorf ME: Cloning of the mouse fusin gene, homologue to a human HIV-1 co-factor. J Immunol 157: 5455-5460, 1996. 